\title{
Idiopathic dilated cardiomyopathy: familial prevalence and HLA distribution
}

\author{
C J McKenna, M B Codd, H A McCann, D D Sugrue
}

\begin{abstract}
Objectives-To compare HLA distribution in familial and non-familial dilated cardiomyopathy, because a serum marker that could identify families at risk of developing dilated cardiomyopathy should be of use in screening for the dis-
\end{abstract} ease.

Patients-100 patients with dilated cardiomyopathy.

Methods-200 first degree relatives from 56 of the proband families were screened for dilated cardiomyopathy by echocardiography. The HLA profile of the patients with dilated cardiomyopathy, as well as of the familial and non-familial subgroups, was compared with that of 9000 normal controls.

Results-The familial prevalence of dilated cardiomyopathy in this patient group was "definite" in 14 of $56(25 \%)$ and "possible" in 25 of $56(45 \%)$. The HLADR4 frequency in the 100 patients with dilated cardiomyopathy was similar to that in the 9000 controls (39\% v 32\%). However, the DR4 subtype was significantly more common in the 25 probands with a familial tendency to dilated cardiomyopathy than in the 31 probands with non-familial dilated cardiomyopathy (68\% v 32\%; $P<0.05)$.

Conclusions-The present finding supports an HLA linked predisposition to familial dilated cardiomyopathy. The HLA type DR4 was significantly more common in familial than in non-familial cases. The DR4 halotype was associated with two thirds of the families at risk for dilated cardiomyopathy.

Mater Misericordiae
Hospital (University
College), Eccles
Street, Dublin 7,
Ireland: Department
of Clinical Cardiology
C J McKenna
H A McCann
D D Sugrue
Department of
Epidemiology and
Biostatistics
M B Codd
Correspondence to:
Dr C J McKenna, Research
Fellow, Division of
Cardiovascular Diseases,
Mayo Clinic, 200 First Street
SW, Rochester, Minnesota
55905, USA.
Accepted for publication
12 February 1997

(Heart 1997;77:549-552)

Keywords: idiopathic dilated cardiomyopathy; HLADR4; screening

Idiopathic dilated cardiomyopathy was defined as global ventricular dilatation with impaired systolic function in the absence of a known cause. ${ }^{1}$ In view of the large amount of ongoing research in the field the definition has recently been expanded to say that dilated cardiomyopathy "may be familial/genetic, viral and/or autoimmune, or alcoholic/toxic". ${ }^{2}$ The most popular causal hypothesis for dilated cardiomyopathy is an autoimmune process initiated by an environmental trigger, for example viral illness, alcohol, or pregnancy. This autoimmune theory has been lent support by an increased frequency of HLA-DR4 and by the presence of circulating cardiac autoantibodies in some patients with dilated cardiomyopathy. ${ }^{34}$

The first prospective studies in which relatives of patients with dilated cardiomyopathy were screened were reported recently and gave a familial prevalence of $20-25 \% .{ }^{56}$ Screening could identify latent forms of the disease which may respond better to treatment, with an improvement in prognosis. The presence of autoantibodies does not adequately distinguish between familial and non-familial dilated cardiomyopathy. ${ }^{78}$ We postulated that the DR4 subtype might identify families at risk of developing the disease.

\section{Methods}

PATIENTS

Patients with dilated cardiomyopathy attending the National Cardiac Centre at the Mater Misericordiae Hospital and their first degree relatives were invited to attend a special clinic. To date 100 probands with dilated cardiomyopathy have been identified. The ultrasound criteria for the diagnosis of dilated cardiomyopathy were a left ventricular ejection fraction less than $50 \%$ combined with a left ventricular end diastolic dimension more than two standard deviations above the mean, corrected for the patient's age and body surface area. ${ }^{9}$ All patients had angiographically normal coronary arteries. Patients with a known cause for heart muscle disease, for example coronary artery disease, hypertension, valvar, and congenital heart disease, as well as infective (myocarditis) and metabolic (thyroid disease) causes were excluded. Patients with a history of possible excessive alcohol consumption were included since they may have a genetic predisposition to develop dilated cardiomyopathy.

HLA typing which included class I (HLA-A, B) and class II (HLA-DR) was performed by the serological microtoxicity method at the National Blood Transfusion Service. The HLA distribution in patients was compared to a reference population of 9000 normal controls. Cross sectional, $M$ mode, and Doppler echocardiographic examinations were performed by a single experienced operator on all patients. In keeping with convention, end systolic and end diastolic cavity dimensions, wall thickness, and fractional shortening were measured by $M$ mode at the papillary muscle level in the parasternal short axis view. 
Table 1 Prevalence of DCM in 56 screened proband families

\begin{tabular}{ll}
\hline Definite DCM & \\
Already diagnosed & $5(9 \%)$ \\
Both echo criteria & $9(16 \%)$ \\
Possible DCM & $9(16 \%)$ \\
One of echo criteria & $6(11 \%)$ \\
Sudden death & $25 / 56(45 \%)$ \\
Total & \\
\hline
\end{tabular}

${ }^{\star}$ Left ventricular ejection fraction $<50 \%$; left ventricular internal diastolic diameter $>2 \mathrm{SD}$ above mean.

\section{FIRST DEGREE RELATIVES}

To date, 200 first degree relatives from 56 proband families have attended for echocardiography. The disease was considered to be familial if at least one first degree relative was diagnosed as having dilated cardiomyopathy, as defined above. If a first degree relative fulfilled only one of the criteria for the diagnosis of dilated cardiomyopathy - that is, a left ventricular ejection below $50 \%$ or a left ventricular end diastolic dimension more than two standard deviations above the mean-they were entered in a "possible" category for further follow up. Any families with a history of premature sudden death (unexplained at an age of less than 50 years) were also included in the "possible" category.

\section{STATISTICS}

Differences between proportions of patients and controls, and familial and non-familial patient subgroups, with regard to HLA distribution were compared using the $\chi^{2}$ test.

\section{Results}

FAMILY SCREENING

A total of 270 first degree relatives from 75 of the 100 patient families were potentially available for screening. Twenty five of the 100 patients either had no family members available for screening or did not wish their relatives to be contacted. So far, 200 of a possible 270 first degree relatives ( $74 \%$ ), from 56 of the 75 proband families $(75 \%)$, have been screened by echocardiography. Those not screened include relatives still to be contacted, non-attenders, and those residing abroad.

Of the 56 families screened, five $(9 \%)$ had a first degree relative already diagnosed as having dilated cardiomyopathy, and a further nine $(16 \%)$ had at least one relative who fulfilled both echocardiographic criteria for dilated cardiomyopathy. Thus the "definite" familial occurrence of dilated cardiomyopathy in this patient group was 14 of $56(25 \%)$. In a further nine families $(16 \%)$, one relative had a "possible" diagnosis of dilated cardiomyopathy, that is, only one of the two echocardiographic criteria was fulfilled. Six families (11\%) gave a history of sudden unexplained death in a first

Table 2 Familial prevalence of DCM in screened first degree relatives: number of relatives/proband family available and screened

\begin{tabular}{lrllll}
\hline & Available & & Screened & & Familial tendency \\
\hline First degree relatives & 270 & $\rightarrow$ & $200(74 \%)$ & $\rightarrow$ & $22(11 \%)$ \\
Proband families & 75 & $\rightarrow$ & $56(75 \%)$ & $\rightarrow$ & $25(45 \%)$ \\
\hline
\end{tabular}

Table 3 HLA distribution in DCM patients compared with controls

\begin{tabular}{lcl}
\hline DR4 frequency & & $P$ value \\
\hline Controls & $2880 / 9000(32 \%)$ & \\
DCM probands & $39 / 100(39 \%)$ & NS \\
DCM probands & $10 / 31(32 \%)$ & NS \\
Non-familial & $17 / 25(68 \%)$ & $<0.05$ \\
Familial & 105 \\
\hline
\end{tabular}

*56 proband families screened by echocardiography.

degree relative and these were recorded as "possible" cases of dilated cardiomyopathy. Thus an additional 15 of $56(27 \%)$ showed a "possible" familial tendency to dilated cardiomyopathy. Four families had more than one first degree relative with a definite or possible diagnosis of dilated cardiomyopathy. Therefore the familial occurrence of dilated cardiomyopathy in this patient group was "definite" in $14(25 \%)$ and "possible" in a total of 25 $(14+11)(45 \%)$ of the 56 proband families screened (tables 1 and 2).

\section{HLA DISTRIBUTION}

The HLA types in the 100 probands were compared with a reference population of 9000 normal controls from the National Transfusion Service. As compared to these controls, the total group of patients with dilated cardiomyopathy showed no significant differences in halotype. However, when only those dilated cardiomyopathy probands with a familial tendency were evaluated, the DR4 subtype was more common than in the whole patient population or in the controls ( $68 \% v 39 \% v 32 \%)$. There was no significant difference in the proportion of all patients with dilated cardiomyopathy who had the DR4 subtype compared with the general population However, within the study group, the proportion of patients with familial dilated cardiomyopathy and HLA-DR4 (17 of $25 ; 68 \%$ ) was greater than the proportion of patients with non-familial dilated cardiomyopathy and HLA-DR4 (10 of 31 ; 32\%) $(\mathrm{P}<0.05$, table 3).

SCREEN DETECTED DILATED CARDIOMYOPATHY Twenty two asymptomatic first degree relatives had abnormal echocardiographic examinations. Eighteen $(82 \%)$ were male and the average age was 40 (range 17-60) years. The ECG was abnormal in only four $(18 \%)$ of these cases, with two patients in atrial fibrillation and two showing non-specific ST changes. Fourteen $(64 \%)$ of the screen detected cases were positive for the DR4 halotype. Thus, as was shown for the probands with familial dilated cardiomyopathy, the screen detected relatives were significantly more likely than controls to have the HLADR4 subtype $(64 \%$ v $32 \% ; \mathrm{P}<0.05)$

\section{Discussion}

In this study of patients with dilated cardiomyopathy, $25 \%$ of cases were definitely familial in nature and $45 \%$ showed a possible familial tendency. This definite familial occurrence is similar to two other recent studies from the 
Table 4 Familial dilated cardiomyopathy (DCM): prospective screening studies (USA, UK, Ireland)

\begin{tabular}{lcll}
\hline & Probands & Relatives & Familial DCM \\
\hline Goerss ${\text { et } a l^{10}}^{10}$ & 95 & 457 & 23 families (24\%) \\
Keeling et $^{6}$ & 40 & 236 & 10 families $(25 \%)$ \\
Present study & 56 & 200 & 14 families (25\%) \\
Total & 191 & 893 & $47(25 \%)$ \\
\hline
\end{tabular}

United Kingdom and the USA (table 4)..$^{610}$ These results may be an underestimate of the true prevalence of familial dilated cardiomyopathy, as not all of the relatives were screened. Somewhat more surprising is the $11 \%$ frequency of premature sudden death. The majority of patients with "idiopathic" life threatening ventricular arrhythmias appear to have an abnormal myocardium on biopsy and these findings may indicate an early form of dilated cardiomyopathy which is too subtle to be detected by usual cardiac investigations. ${ }^{11}$ The HLA type DR4 was significantly more common in patients with familial dilated cardiomyopathy than in those with non-familial dilated cardiomyopathy or in comparison to the total patient group. More importantly, HLA-DR4 was associated with two thirds of the families at risk for dilated cardiomyopathy. The asymptomatic, screen detected relatives with dilated cardiomyopathy were also significantly more likely than controls to have the DR4 halotype. DR4 has been associated with other autoimmune diseases. The present finding supports an HLA linked predisposition to the development of familial dilated cardiomyopathy.

Anderson et al in 1984 were the first to report a HLA link in patients with dilated cardiomyopathy. ${ }^{3}$ They found that the HLA types DR4 and B27 were commoner in patients than controls. The halotype frequency of B27 was $14.5 \%$ in patients with dilated cardiomyopathy compared with $3.3 \%$ in controls, while the frequency of DR 4 was $54 \%$ in dilated cardiomyopathy and $32 \%$ in controls $(P=0.001$ and 0.02 , respectively). The HLA-DR4 association with dilated cardiomyopathy has since been confirmed by many other studies ${ }^{12}{ }^{17}$ and refuted by one. ${ }^{18}$ The latter was a retrospective look at a particular subset of patients who underwent cardiac transplantation. It is of interest to note that patients undergoing heart transplantation have less cellular rejection and increased long term survival if they are HLA-DR matched with the donor heart. ${ }^{19}$ This matching process may be of particular importance in patients with familial dilated cardiomyopathy who are being considered for transplantation.

The search for humoral markers of autoimmunity in patients with dilated cardiomyopathy has been going on for the last 20 years. Natural killer leucocytes are known to be important in host immune surveillance and mediate resistance to viral infections. Natural killer cell deficiency has been shown to be a disease marker for dilated cardiomyopathy, and this subgroup of patients with dilated cardiomyopathy was distinguished from others by an increase in specific HLA halotypes. ${ }^{20}$ An increase in $\mathrm{T}$ helper: $\mathrm{T}$ suppressor cell ratios has been variably demonstrated in dilated cardiomyopathy and is a typical feature of autoimmune disease..$^{21}$ Nearly half of patients with dilated cardiomyopathy have evidence for a chronic autoimmune process by immunohistochemical analysis of their cardiac biopsies. ${ }^{22}$ Circulating organ specific cardiac autoantibodies have been found in some patients with dilated cardiomyopathy and have been detected more often in those patients with recent onset of disease. ${ }^{47}$ This also suggests a cell mediated autoimmune mechanism. The reason for the presence of serological markers in only some patients with dilated cardiomyopathy is postulated to be due to either different aetiologies or a reduction in antibody level with disease progression.

The evidence suggests that dilated cardiomyopathy can be associated with the host's immune response and the genetic factors that control this response. It is conceivable that familial dilated cardiomyopathy is the subgroup of patients with the disease with a defect in their immune response. This defect may then manifest itself as an autoantibody directed against the myocardium following an environmental stimulus, for example a viral illness. This theory is lent support by correlations between specific $\mathrm{T}$ cell gene alleles ${ }^{23}$ and HLA types ${ }^{17}$ and the presence of autoantibodies in familial cardiomyopathy. Organ specific cardiac antibodies have also been detected in symptom-free relatives of patients with dilated cardiomyopathy. ${ }^{7}$

Relatives of dilated cardiomyopathy patients with the DR4 subtype are at particular risk of developing the disease and should probably be screened regularly by echocardiography, as well as counselled regarding potential lifestyle and pharmacological interventions which might alter the natural history of the disease. Obviously, some relatives with a normal echo at a single screening may later develop dilated cardiomyopathy. Only an ongoing prospective study screening all available first degree relatives on a regular basis would determine the true prevalence of familial dilated cardiomyopathy and the usefulness of markers such HLA typing in identifying those families at greatest risk. When sufficient asymptomatic screen detected relatives with dilated cardiomyopathy are identified, randomised trials of ACE inhibition or immunotherapy to slow disease progression should be initiated.

This study was supported in part by a grant from the Mater Hospital Foundation.

1 Report of the WHO/ISFC task force on the definition and classification of cardiomyopathies. Br Heart $\mathcal{F} 1980 ; 44$ 672-3.

2 Report of the WHO/ISFC task force on the definition and classification of cardiomyopathies. Circulation 1996;93. 841-2.

3 Anderson JL, Carlquist JF, Lutz JR, DeWitt CW, Hammond EH. HLA A, B and DR typing in idiopathic dilated cardiomyopathy: dilated cardiomyopathy: a search for immune response
factors. Am $\mathcal{\text { Cardiol }}$ 1984;53:1326-30.

4 Caforio ALP, Bonifacio E, Stewart JT, et al. Novel organspecific autoantibodies in dilated cardiomyopathy. $f \mathrm{Am}$ ol 1990;15:1527-34.

5 Michels VV, Moll PP, Miller FA, et al. The frequency of familial dilated cardiomyopathy in a series of patients 
with idiopathic dilated cardiomyopathy. $N$ Engl $\mathcal{F} \mathrm{Med}$ 1992;326:77-82.

6 Keeling PJ, Gang Y, Smith G, et al. Familial dilated cardiomyopathy in the United Kingdom. Br Heart $\mathcal{f} 1995$ 73:417-2

7 Caforio ALP, Keeling PJ, Zachara E, et al. Evidence from familial studies for autoimmunity in dilated cardiomyopathy. Lancet 1994;344:773-7.

8 Michels VV, Moll PP, Rodeheffer RJ, et al. Circulating heart autoantibodies in familial as compared with nonfamilial idiopathic dilated cardiomyopathy. Mayo Clin Proc 1994;69:24-7.

9 Henry WL, Gardin JM, Ware JH. Echocardiographic measurements in normal subjects from infancy to old age. Circulation 1980;62:1054-61.

10 Goerss JB, Michels VV, Burnett JB, et al. Frequency of familial dilated cardiomyopathy. Eur Heart $f$ 1995;16: $2-4 S$.

11 Sugrue DD, Holmes DR, Gersh BJ, et al. Cardiac histologic findings in patients with life-threatening ventricular logic findings in patients with life-threatening ventricular
arrhythmias of unknown origin. $f \mathrm{Am}$ Coll Cardiol 1984; arrhythmias

12 Limas CJ, Limas C. HLA antigens in idiopathic dilated cardiomyopathy. Br Heart $\mathcal{F}$ 1989;62:379-83.

13 Arbustini E, Gavazzi A, Pozzi R, et al. The morphologic spectrum of dilated cardiomyopathy and its relation to immune-response genes. Am $\mathcal{F}$ Cardiol 1989;64:991-5.

14 Carlquist JF, Menlove RL, Murray MB, O'Connell JB, Anderson JL. HLA class II (DR and DQ) antigen associations in idiopathic dilated cardiomyopathy. Circulation 1991;83:515-22.

15 Martinetti M, Dugoujon JM, Caforio ALP, et al. HLA and immunoglobulin polymorphisms in idiopathic dilated cardiomyopathy. Hum Immunol 1992;35:193-9.

16 Bland NK, Schwimmbeck PL, Kuhl U, Enczmann J, Wernet P. HLA DR association in myocarditis and dilated cardiomyopathy. Circulation 1992;86-I:794.

17 Limas C, Limas CJ, Boudoulas H, et al. Anti- $\beta$-receptor antibodies in familial cardiomyopathy: correlation with HLA-DR and HLA-DQ gene polymorphism. Am Heart f 1994;127:382-6

18 Grant SCD, Sheldon S, Dyer PA, Levy RD, Brooks NH. Do specific HIA antigens predispose to ischaemic heart disease or idiopathic dilated cardiomyopathy? Br Heart $\mathcal{F}$ 1994;71:76-8.

19 Smith JD, Rose ML, Pomerance A, Burke M, Yacoub MH. Reduction in cellular rejection and increase in longerReduction in matching. Lancet 1995;346:1318-22.

20 Anderson JL, Carlquist JF, Hammond EH. Deficient natural killer cell activity in patients with idiopathic dilated ural killer cell activity in patients with idiopathic dilated

21 Huber KC, Gersh BJ, Sugrue DD, et al. T-lymphocyte subsets in patients with idiopathic dilated cardiomyopathy. Int $\mathcal{F}$ Cardiol 1989;22:59-66.

22 Herskowitz A, Ahmed-Ansari A, Neumann DA, et al. Induction of major histocompatibility antigens within the myocardium of patients with active myocarditis: a nonhistologic marker of myocarditis. F Am Coll Cardiol 1990; 15:624-32.

23 Limas CL, Limas CJ, Boudoulas H, et al. T-cell receptor gene polymorphisms in familial cardiomyopathy: correlation with anti- $\beta$-receptor autoantibodies. Am Heart $\mathscr{f}$ 1992;124:1258-63. 